\title{
Primary Care Provider Perceptions of Colorectal Cancer Screening Barriers: Implications for Designing Quality Improvement Interventions
}

\author{
Jennifer M. Weiss, ${ }^{1,2,3}$ Perry J. Pickhardt, ${ }^{4}$ Jessica R. Schumacher, ${ }^{5}$ Aaron Potvien, ${ }^{6}$ \\ David H. Kim, ${ }^{4}$ Patrick R. Pfau, ${ }^{1,2}$ Elizabeth A. Jacobs, ${ }^{2}$ and Maureen A. Smith ${ }^{3,5,6,7}$ \\ ${ }^{1}$ Division of Gastroenterology and Hepatology, University of Wisconsin School of Medicine and Public Health, Madison, WI, USA \\ ${ }^{2}$ Department of Medicine, University of Wisconsin School of Medicine and Public Health, Madison, WI, USA \\ ${ }^{3}$ University of Wisconsin Carbone Cancer Center, Madison, WI, USA \\ ${ }^{4}$ Department of Radiology, University of Wisconsin School of Medicine and Public Health, Madison, WI, USA \\ ${ }^{5}$ Department of Surgery, University of Wisconsin School of Medicine and Public Health, Madison, WI, USA \\ ${ }^{6}$ Department of Population Health Sciences, University of Wisconsin School of Medicine and Public Health, Madison, WI, USA \\ ${ }^{7}$ Department of Family Medicine, University of Wisconsin School of Medicine and Public Health, Madison, WI, USA
}

Correspondence should be addressed to Jennifer M. Weiss; jmw@medicine.wisc.edu

Received 21 August 2016; Revised 10 December 2016; Accepted 20 December 2016; Published 10 January 2017

Academic Editor: Leticia Moreira

Copyright ( $(2017$ Jennifer M. Weiss et al. This is an open access article distributed under the Creative Commons Attribution License, which permits unrestricted use, distribution, and reproduction in any medium, provided the original work is properly cited.

\begin{abstract}
Aims. Colorectal cancer (CRC) screening is underutilized. Increasing CRC screening rates requires interventions targeting multiple barriers at each level of the healthcare organization (patient, provider, and system). We examined groups of primary care providers (PCPs) based on perceptions of screening barriers and the relationship to CRC screening rates to inform approaches for conducting barrier assessments prior to designing and implementing quality improvement interventions. Methods. We conducted a retrospective cohort study linking EHR and survey data. PCPs with complete survey responses for questions addressing CRC screening barriers were included ( $N=166$ PCPs; 39,430 patients eligible for CRC screening). Cluster analysis identified groups of PCPs. Multivariate logistic regression estimated odds ratios and $95 \%$ confidence intervals for predictors of membership in one of the PCP groups. Results. We found two distinct groups: (1) PCPs identifying multiple barriers to CRC screening at patient, provider, and system levels $(N=75)$ and (2) PCPs identifying no major barriers to screening $(N=91)$. PCPs in the top half of CRC screening performance were more likely to identify multiple barriers than the bottom performers (OR, 4.14; 95\% CI, 2.43-7.08). Conclusions. High-performing PCPs can more effectively identify CRC screening barriers. Targeting high-performers when conducting a barrier assessment is a novel approach to assist in designing quality improvement interventions for CRC screening.
\end{abstract}

\section{Introduction}

Despite recent improvements in colorectal cancer (CRC) incidence and mortality, CRC remains the second leading cause of cancer-related death for men and women in the United States [1]. If screening modalities were optimally employed, CRC mortality would be largely preventable [29]. Although CRC screening is strongly endorsed by multiple professional societies and achievable using a variety of methods, national rates remain suboptimal, with only twothirds of eligible individuals undergoing screening [10-12].

Previous efforts to improve CRC screening rates in the United States yielded an overall increase from $50 \%$ to $65 \%$ over the past decade. However, rates have reached a plateau [13]. Evidence suggests targeting interventions to specifically identified barriers is likely to change practice [14]. This is critical to achieving further improvements in CRC screening rates, given the number and complexity of potential CRC 
screening barriers. However, as stated in a Cochrane review, "we do not yet know the most effective ways to identify barriers, to pick out from amongst all the barriers those that are most important to address, or how to select interventions likely to overcome them" [14].

Primary care providers (PCPs) are a logical source of information about barriers to CRC screening due to their integral role in cancer prevention. PCP recommendation is one of the strongest predictors of screening utilization, even with insurance disparities [15-19]. Yet it is not known which PCPs are best positioned to identify key CRC screening barriers in a health system. It is possible that targeting specific subgroups of PCPs (e.g., low- or high-performers) might yield the most comprehensive barrier assessment. In this study, we examine PCPs' identification of major barriers to CRC screening at each level of the healthcare system, determine if distinct groups of PCPs exist, and examine predictors of membership in these groups. Our results provide valuable information for determining which PCPs can reliably identify CRC screening barriers, with important implications for conducting barrier assessments prior to designing quality improvement interventions.

\section{Materials and Methods}

2.1. Study Design. The study was conducted in one of the 12 largest multispecialty physician groups in the United States. This group has approximately 1.7 million ambulatory visits per year, delivered by over 300 PCPs in more than 40 multispecialty and community-based primary care clinic sites.

A survey of CRC screening beliefs and practices was mailed to all PCPs within the physician group in February 2010. The goal was to gather data on the current landscape of CRC screening practices in the participating healthcare system prior to designing and implementing system-wide quality improvement interventions. The survey was based on the National Cancer Institute Survey of Colorectal Cancer Screening Practices, developed in collaboration with the CDC and Centers for Medicare and Medicaid Services, and has been used extensively in prior research [15, 20-22]. Survey items were divided into four sections: (1) cancer screening beliefs and practices; (2) attitudes toward CRC screening; (3) CRC screening modalities; and (4) provider characteristics. The overall survey response rate was 70\% $(N=$ $226 / 322$ ). We present an analysis of a specific subset of survey items that assessed the importance of perceived barriers to CRC screening at the patient, provider, and system levels and restricted the sample to respondents who completed the entire subset of interest $(N=166 / 226), 73 \%$ of the survey responders. Survey responses were linked to patient panel, provider, and clinic level characteristics obtained from the electronic health record (EHR). This study was approved by the Institutional Review Board at the University of Wisconsin-Madison.

2.2. Survey Variables. Survey items assessed PCP perceptions of barriers to CRC screening at patient, provider, and system levels. Responses were dichotomized as "major barrier" or "minor/not a barrier." Patient-level barriers included fear of finding cancer, belief that screening is not effective, embarrassment or anxiety about screening tests, lack of awareness of screening or perception of CRC as a nonserious health threat, fear of an invasive test, and concern about tolerating colonoscopy bowel prep. Provider-level barriers included the perception that PCPs do not routinely recommend screening to their patients and lack of time in clinic to discuss screening. System-level barriers included long wait times between ordering and scheduling a test, financial cost to patients, shortage of trained providers to conduct screening, and lack of a system for identifying patients eligible for screening. Two additional questions were included to assess provider and system-level barriers. The provider barrier question asked respondents if they had a method to identify which patients were in need of CRC screening exams. Answer choices were yes (coded as "minor/not a barrier") or no (coded as "major barrier"). The system barrier question instructed respondents to "comment on the current capacity of facilities and personnel in your organization to meet the demand for performing colonoscopy." Answer choices were "more than enough," "just about right," "inadequate," and "don't know." Responses were dichotomized with "inadequate" considered a "major barrier," and "more than enough/just about right/don't know" coded as "minor/not a barrier."

2.3. Patient, Provider, and Clinic Characteristics. Sample characteristics were obtained from the EHR. Patient variables included age, gender, race, marital status, primary language, insurance coverage, and comorbidities. A healthcare resource utilization score was calculated for each patient using Ambulatory Care Groups (ACG) based on outpatient and inpatient diagnoses from 12 months prior to survey administration [23, 24]. Provider variables included gender, specialty (Internal Medicine/Family Medicine), years in practice, CRC screening rates in 2009, and size of patient panel eligible for CRC screening. Clinic variables included clinic management and number of providers within the clinic. Clinic distance to the nearest colonoscopy facility was calculated using geographical software. Patients were assigned to PCPs using the plurality provider algorithm described by Pham et al. [25]. PCPs were assigned to clinics by the clinic at which the provider billed the majority of their Evaluation \& Management (E\&M) visits in 2009.

2.4. Identification of Screen-Eligible Population. We used EHR data to identify the pool of patients eligible for CRC screening in 2009 based on Healthcare Effectiveness Data and Information Set (HEDIS) metrics [26]. Adults aged 50-75 years were included if they were "currently managed" by the physician group. The definition of "currently managed" has been previously published [20]. Patients were excluded if they had a total colectomy based on ICD-9 codes and CPT codes.

2.5. Identification of CRC Screening Completion. Completion of CRC screening was defined as (a) fecal occult blood test (FOBT) in the prior 12 months, (b) flexible sigmoidoscopy, double contrast barium enema, or CT colonography in the past 5 years [11], or (c) colonoscopy in the past 10 years 
determined by HEDIS codes [26]. All PCPs in the physician group have access to colonoscopy and CT colonography, and most local third party payers cover CT colonography as a CRC screening option at the participating institution [27].

2.6. Statistical Analysis. Our primary goal was to determine if distinct categories of PCPs exist based on perceived CRC screening barriers. Cluster analysis was used to organize PCPs into meaningful structures based on survey responses [28]. Final identification of cluster groups was based on hierarchical cluster analysis using average linkage. Hierarchical clustering allows smaller clusters to be nested within larger ones reflecting a gradation of survey responses; average linkage allows clusters to be hierarchically related without depending on prior knowledge that the clusters resemble chains (single linkage) or are spatially compact (complete linkage) [29].

We compared the frequency of patient, provider, and clinic variables for the PCP cluster groups using $\chi^{2}$ tests for categorical variables and two-way analysis of variance tests for continuous variables. Multivariate logistic regression with robust estimation of standard errors and clustering at the clinic level was performed using the logit procedure in Stata to obtain odds ratios and 95\% confidence intervals for provider and clinic level predictors of membership in one of the PCP groups. Analyses were conducted with Stata 12.0 (StataCorp, College Station, TX) and SAS 9.3 (SAS Institute, Cary, NC) software. All tests of significance used two-sided $p$ values at the $p<0.05$ level.

\section{Results}

3.1. Distribution of Survey Responses. Cluster analysis identified two groups of PCPs based on perceived barriers to CRC screening at patient, provider, and system levels: (1) PCPs who perceived multiple major barriers to CRC screening $(N=$ 75) and (2) PCPs who perceived no major barriers to CRC screening $(N=91)$. Figure 1 shows the percent of PCPs in each group who perceived each patient, provider, and systemlevel barrier as a major barrier.

3.2. Sample Characteristics. Overall, the 166 PCPs included in this analysis worked at 24 primary care clinics and cared for 39,430 patients eligible for CRC screening, according to the methodology previously described. The majority of patients were 50-60 years old, White, married, primarily English speaking, and covered by commercial insurance (Table 1). Of the 166 PCPs, 52\% were female, over half practiced Internal Medicine (52\%), and two-thirds had practiced $>10$ years. The average number of patients eligible for CRC screening in a provider's panel was 238. Two-thirds of the primary care clinics were physician-owned with an average distance of 7.6 miles to the nearest colonoscopy facility.

PCPs who perceived multiple barriers to CRC screening $(N=75)$ cared for 26,420 patients eligible for screening and worked at 14 different primary care clinics. Comparatively, PCPs who perceived no major barriers to CRC screening $(N=91)$ cared for 13,010 patients eligible for screening and worked at 10 different primary care clinics. The patients assigned to PCPs who perceived multiple barriers were more often female ( $61 \%$ versus $48 \%, p<0.001)$, spoke English as a primary language ( $92 \%$ versus $89 \%, p<0.001$ ), and had more commercial insurance coverage $(67 \%$ versus $63 \%, p<0.001)$. There was no significant difference between the percent of patients with congestive heart failure and diabetes mellitus or the average ACG resource utilization score between the patients assigned to the two groups. Providers who perceived multiple barriers were more often female (61\% versus $45 \%$, $p=0.043$ ), had a higher CRC screening rate on average (66\% versus 59\%, $p=0.013$ ), and on average had a larger patient panel eligible for CRC screening compared to the PCPs who perceived no major barriers (250 versus 91 patients, $p<0.001)$. There was no significant difference in clinic characteristics between the two groups of PCPs.

3.3. Predictors of Membership in PCP Groups. In the adjusted model with provider and clinic characteristics, the most significant predictor of membership in one of the PCP groups was provider CRC screening rate (Table 2). PCPs identifying multiple barriers were more likely to be in the top half in terms of CRC screening performance (OR, 4.14; 95\% CI, 2.43-7.08). The mean CRC screening rate for the top performers compared to the bottom performers was $75 \%$ versus 49\% (data not shown). PCP gender, specialty, and years in practice were not significant predictors of membership in a PCP group.

\section{Discussion}

Our study identified two distinct groups of primary care providers for assistance with CRC screening barrier assessment: (1) PCPs who perceive multiple major barriers to CRC screening and (2) PCPs who perceive no major barriers. The PCPs identifying multiple barriers were four times as likely to be in the top half in terms of CRC screening performance compared to PCPs identifying no major barriers. We hypothesize that high-performers were able to identify barriers at multiple levels of the healthcare system because they are more actively engaged in the CRC screening process and therefore encounter and are attuned to more barriers. This is a critical finding, as the identification of key barriers to screening may allow for the development of targeted interventions to improve CRC screening at a time when rates have plateaued [13].

We found that the PCPs who identified more barriers to CRC screening accurately assessed the climate of our healthcare system at the time of the survey. At the time of survey administration there was a shortage of gastroenterologists in our healthcare system to perform colonoscopies, inadequate capacity to meet the demand for colonoscopies, and very long wait times ( $>1$ year) between ordering and scheduling the exam. These PCPs also identified lack of a system for identifying patients eligible for screening as a major barrier. Although all clinics and PCPs used the same EHR, there was no standard alert at the time of the survey. In response to the survey data, more gastroenterologists were hired to address these issues, significant changes were made to the scheduling process, and the wait time was successfully decreased to $<3$ 


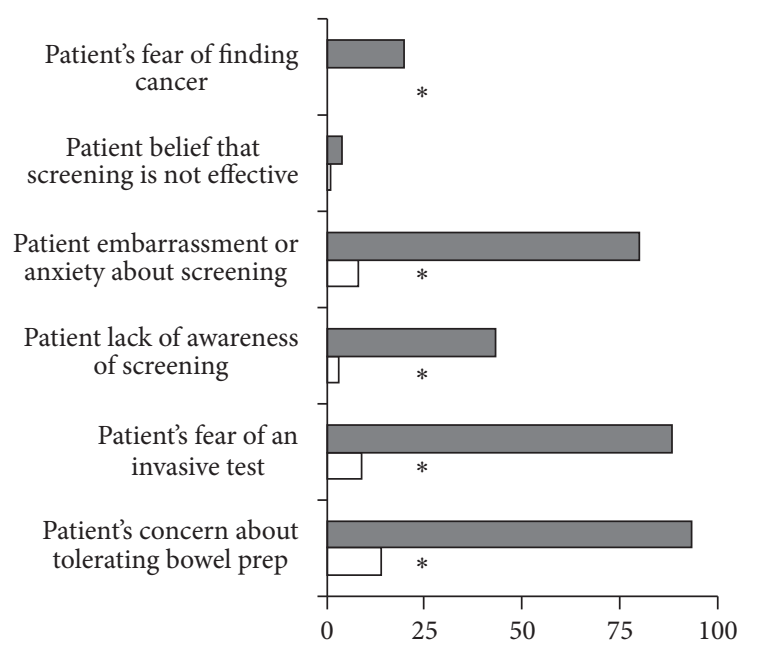

(\%)

PCP Group 1 - Multiple Barriers $(N=75)$

$\square$ PCP Group 2 - No Major

Barriers $(N=91)$

${ }^{*} p<0.0001$

(a) Patient-level barriers

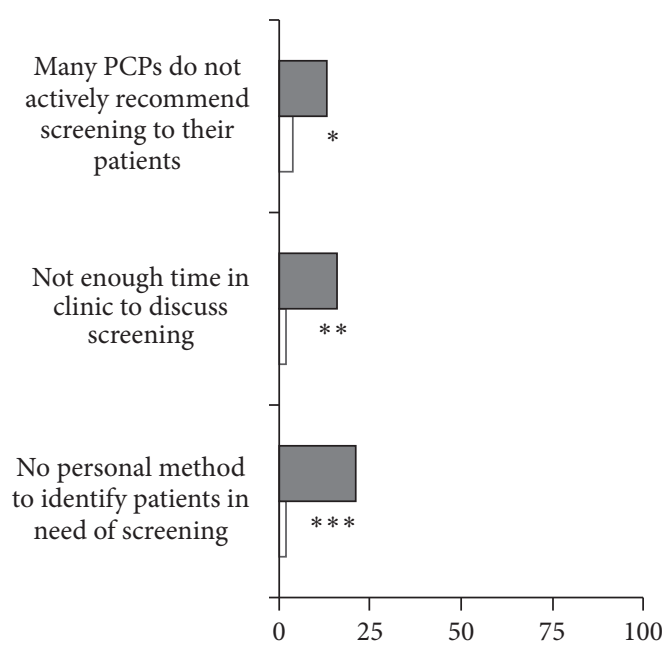

(\%)

口 PCP Group 1 - Multiple Barriers $(N=75)$

$\square$ PCP Group 2 - No Barriers $(N=91)$

${ }^{*} p<0.05$

${ }^{* * *} p=0.001$

${ }^{* * *} p<0.0001$

(b) Provider-level barriers

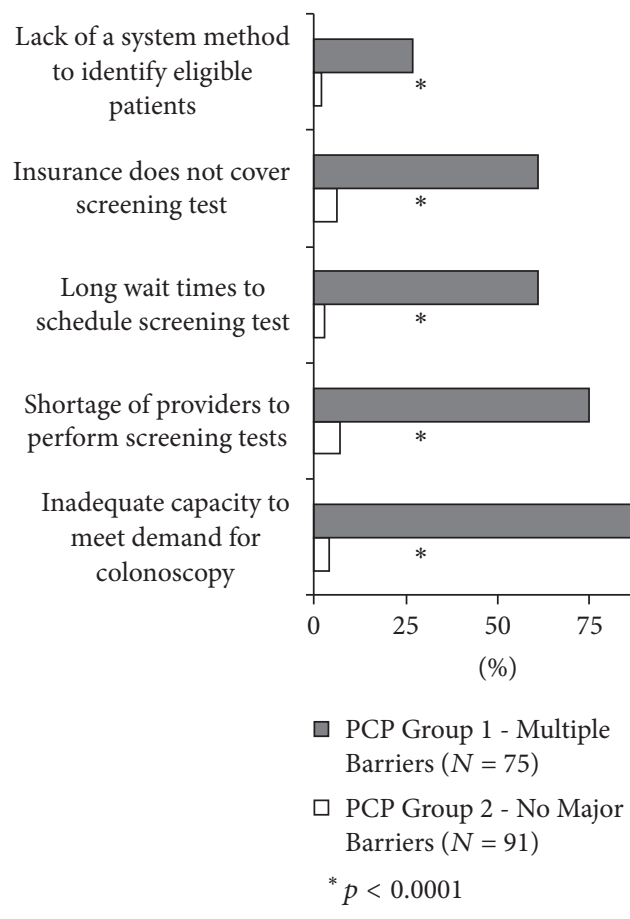

(c) System-level barriers

FIGURE 1: Distribution of survey responses across the two groups of providers for (a) perceived patient-level barriers, (b) perceived providerlevel barriers, and (c) perceived system-level barriers. 
TABLE 1: Sample characteristics of patients, providers, and clinics (overall and by provider perceptions of barriers).

\begin{tabular}{|c|c|c|c|c|}
\hline & Overall & $\begin{array}{l}\text { Multiple perceived } \\
\text { barriers to CRC } \\
\text { screening }\end{array}$ & $\begin{array}{l}\text { No perceived barriers } \\
\text { to CRC screening }\end{array}$ & $p$ value \\
\hline Patient characteristics & $N=39,430$ & $N=26,420$ & $N=13,010$ & \\
\hline \multicolumn{5}{|l|}{ Age, $\%$} \\
\hline $50-54$ & 27 & 27 & 27 & \multirow{5}{*}{$<0.001$} \\
\hline $55-59$ & 27 & 27 & 26 & \\
\hline $60-64$ & 21 & 21 & 21 & \\
\hline $65-69$ & 14 & 14 & 14 & \\
\hline $70-75$ & 11 & 11 & 12 & \\
\hline \multicolumn{4}{|l|}{ Gender, \% } & \multirow{2}{*}{$<0.001$} \\
\hline Female & 56 & 61 & 48 & \\
\hline \multicolumn{4}{|l|}{ Race, $\%$} & \multirow[t]{2}{*}{0.046} \\
\hline White & 93 & 93 & 92 & \\
\hline \multicolumn{4}{|l|}{ Marital status, $\%$} & \multirow{2}{*}{0.004} \\
\hline Married & 71 & 70 & 72 & \\
\hline \multicolumn{5}{|l|}{ Language, $\%$} \\
\hline English (as primary language) & 91 & 92 & 89 & \multirow{3}{*}{$<0.001$} \\
\hline Primarily non-English & 1 & 1 & 1 & \\
\hline Unknown & 8 & 7 & 10 & \\
\hline \multicolumn{5}{|l|}{ Insurance, \% } \\
\hline Commercial & 66 & 67 & 63 & \multirow{4}{*}{$<0.001$} \\
\hline Medicare & 24 & 24 & 25 & \\
\hline Medicaid or uninsured & 2 & 2 & 2 & \\
\hline Missing & 9 & 8 & 10 & \\
\hline \multicolumn{5}{|l|}{ Comorbidities, $\%$} \\
\hline Congestive heart failure & 1 & 1 & 1 & 0.835 \\
\hline Diabetes mellitus & 9 & 9 & 10 & 0.437 \\
\hline Hypertension & 36 & 36 & 35 & 0.005 \\
\hline ACG resource utilization score (mean, SD) & $0.58(0.42)$ & $0.58(0.42)$ & $0.58(0.43)$ & 0.537 \\
\hline Primary care provider characteristics & $N=166$ & $N=75$ & $N=91$ & \\
\hline \multicolumn{5}{|l|}{ Age, $\%$} \\
\hline $30-39$ & 24 & 20 & 27 & \multirow{5}{*}{0.470} \\
\hline $40-49$ & 26 & 29 & 23 & \\
\hline $50-59$ & 25 & 28 & 22 & \\
\hline $60-69$ & 9 & 11 & 8 & \\
\hline Missing & 16 & 12 & 20 & \\
\hline \multicolumn{4}{|l|}{ Gender, \% } & \multirow{2}{*}{0.043} \\
\hline Female & 52 & 61 & 45 & \\
\hline \multicolumn{5}{|l|}{ Specialty, \% } \\
\hline Internal medicine & 52 & 53 & 52 & \multirow[t]{2}{*}{0.133} \\
\hline Family medicine & 48 & 47 & 48 & \\
\hline \multicolumn{5}{|l|}{ Years in practice, $\%$} \\
\hline$<10$ yrs & 18 & 15 & 21 & \multirow{4}{*}{0.358} \\
\hline $10-20 \mathrm{yrs}$ & 31 & 33 & 30 & \\
\hline$>20 \mathrm{yrs}$ & 35 & 40 & 31 & \\
\hline Missing & 16 & 12 & 18 & \\
\hline $\begin{array}{l}\text { Average CRC screening rate }(\%) \text { in the year prior to } \\
\text { survey administration (mean, SD) }\end{array}$ & $62(17.5)$ & $66(15.7)$ & $59(18.4)$ & 0.013 \\
\hline $\begin{array}{l}\text { Number of patients eligible for CRC screening in a } \\
\text { provider's panel (mean, SD) }\end{array}$ & $238(232)$ & $250(182)$ & $91(128)$ & $<0.001$ \\
\hline
\end{tabular}


TABLE 1: Continued.

\begin{tabular}{lccc}
\hline & Overall & $\begin{array}{c}\text { Multiple perceived } \\
\text { barriers to CRC } \\
\text { screening }\end{array}$ & $\begin{array}{c}\text { No perceived barriers } \\
\text { to CRC screening }\end{array}$ \\
\hline $\begin{array}{l}\text { Primary care clinic characteristics } \\
\text { Clinic management, } \%\end{array}$ & $N=24$ & $N=14$ & $N=10$ \\
$\quad \begin{array}{l}\text { Physician-owned } \\
\text { Hospital-owned }\end{array}$ & 67 & 64 & 70 \\
$\begin{array}{l}\text { Distance to nearest colonoscopy center in miles, (mean, } \\
\text { SD) }\end{array}$ & $7.6(5.6)$ & 36 & 30 \\
Number of providers within the clinic, (mean, SD) & $6.9(4.9)$ & $8.1(3.8)$ & $8.5(7.7)$ \\
\hline
\end{tabular}

$\mathrm{SD}=$ standard deviation, $\mathrm{ACG}=$ ambulatory care group, and $\mathrm{CRC}=$ colorectal cancer.

TABLE 2: Adjusted odds ratios and 95\% confidence intervals for provider and clinic predictors of membership in PCP group perceiving multiple barriers $(N=166)$.

\begin{tabular}{|c|c|c|c|}
\hline & OR & $95 \%$ CI & $p$ value \\
\hline \multicolumn{4}{|l|}{ Primary care provider characteristics } \\
\hline \multicolumn{4}{|l|}{ Gender } \\
\hline Male & (ref) & & \\
\hline Female & 2.13 & $0.88,5.13$ & 0.090 \\
\hline \multicolumn{4}{|l|}{ Specialty } \\
\hline Family medicine & (ref) & & \\
\hline Internal medicine & 0.69 & $0.19,2.37$ & 0.552 \\
\hline \multicolumn{4}{|l|}{ Years in practice } \\
\hline$<10 \mathrm{yrs}$ & (ref) & & \\
\hline $10-20$ yrs & 1.12 & $0.53,2.35$ & 0.764 \\
\hline$>20 \mathrm{yrs}$ & 1.24 & $0.52,2.92$ & 0.626 \\
\hline Missing & 0.69 & $0.24,2.00$ & 0.498 \\
\hline \multicolumn{4}{|l|}{ CRC screening rate } \\
\hline Bottom half & (ref) & & \\
\hline Top half & 4.14 & $2.43,7.08$ & $<0.0001$ \\
\hline \multicolumn{4}{|l|}{ Primary care clinic characteristics } \\
\hline \multicolumn{4}{|l|}{ Clinic management } \\
\hline Hospital-owned & (ref) & & \\
\hline Physician-owned & 0.53 & $0.18,1.57$ & 0.253 \\
\hline Distance to nearest colonoscopy center in miles & 0.97 & $0.87,1.07$ & 0.520 \\
\hline Number of providers within the clinic & 0.95 & $0.90,0.99$ & 0.044 \\
\hline
\end{tabular}

$\mathrm{OR}=$ odds ratio, $\mathrm{CI}=$ confidence interval, $\mathrm{ACG}=$ ambulatory care group, and $\mathrm{CRC}=$ colorectal cancer.

months. In addition, a system-wide health maintenance alert was developed for patients overdue for CRC screening and was implemented for all clinics and PCPs through the EHR.

Interestingly, few PCPs in both groups felt that PCPs not actively recommending screening to their patients was a major barrier. This is despite the fact that the large majority of patients who are not current with CRC screening, 94\% of those are over 50 years old [16] and $84 \%$ of those over 65 years old [17], list no physician recommendation as a major reason. This may be due to the fact that PCPs are not aware of their degree of influence on a patient's decision to be screened or they think that the majority of PCPs are recommending CRC screening to all eligible patients. Also of interest is that PCP years in practice did not predict membership in a PCP group. This is contrary to some studies that suggest that providers who have been in practice longer may be less likely to deliver high-quality care, possibly due to out of date information or rapidly changing guidelines $[30,31]$.

There is significant evidence that interventions tailored to specific barriers can improve care delivery. In a large metaanalysis, Baker et al. [14] compared interventions designed to improve receipt of preventive care that were either tailored or not tailored to address identified barriers and compared these groups to a no-intervention control group. They concluded that interventions tailored to prospectively identified barriers are more likely to improve practice. Tailoring interventions to increase CRC screening is critical, due to the complexity of barriers that results from multiple available CRC screening modalities, the number of providers required for the process, and the various locations where screening can be performed. 
Baker et al. [14] also noted that the methods used to identify barriers varied widely between the studies; therefore, the best methods for identifying barriers require further research. It is common practice to call upon high-performing providers and healthcare systems to share their "best practices" so that lower-performers can learn how to improve their CRC screening rates [32]. In addition, we often see low-performing providers interviewed or surveyed about the barriers that are preventing them from being a "high-performer." Our results suggest that lower-performing providers are less likely to identify barriers at different levels within the healthcare system. The results also suggest that initial barrier assessments should target high-performing providers to identify pertinent barriers and possible facilitators to assist in the design and implementation of interventions to improve CRC screening rates.

There are limitations to this study. First, we report findings from a large academic physician group, which could impact generalizability. However, large multispecialty systems are quickly becoming a preferred way to provide high-quality health care and are therefore critical to the understanding of modern health care delivery [33]. Second, our nearly all-white patient population may limit potential generalizability to healthcare systems with a more diverse patient population. Third, the survey reports perceptions of barriers to CRC screening in our healthcare system in 20092010. While this was more than five years ago, there is no reason to believe that perceptions of barriers have fundamentally shifted since that time. In addition, our study shows that high-performers are able to more accurately identify CRC screening barriers within a healthcare system and this same concept can be applied to other systems where the exact details of the barriers may be different. Fourth, there are inherent selection biases when relying on survey data, such as nonresponse bias [34-36]. This impact is likely minimal due to our high response rate. Typical survey response rates for healthcare providers are well below 50\% [37]. Our overall response rate was $70 \%$; the sample for this study included providers with complete responses to the questions of interest which is $51 \%$ of the overall sample. Fifth, a number of our variables rely on the EHR, which could result in missing data and possible misclassification of a completed screening test. However, this is unlikely to result in a systematic bias across clinical settings since all clinics used a fully integrated EHR that has been populated with all data since 1991 (including scanned documents that were manually reviewed to assess completion of CRC screening outside the system). Finally, our cluster analysis used dichotomized survey responses (major barrier versus minor/not a barrier) which results in a loss of some of the finer details in the survey data. However, this was necessary due to the sample size.

\section{Conclusions}

Multiple healthcare organizations across the country have signed the American Cancer Society call for screening 80\% of eligible patients for colorectal cancer by 2018. In order to reach this goal, large initiatives will be formed. In this study, we identified two groups of PCPs: (1) PCPs who perceive multiple major barriers to CRC screening and (2) PCPs who perceive no major barriers. PCPs identifying multiple barriers were more likely to be in the top half in terms of CRC screening performance, suggesting that highperformers of quality metrics of interest should be targeted as the source for effective barrier identification prior to the design and implementation of interventions. Future research will involve determining if the impact and sustainability of these interventions are enhanced for providers who identified the barriers or if the impact is the same for all PCPs.

\section{Disclosure}

The funding sources had no role in the design and conduct of the study; collection, management, analysis, and interpretation of the data; preparation, review, or approval of the manuscript; or the decision to submit this manuscript for publication.

\section{Competing Interests}

Perry J. Pickhardt, M.D., is a cofounder of VirtuoCTC and shareholder in SHINE and Cellectar. David H. Kim, M.D., is a cofounder of VirtuoCTC, consultant for Viatronix, on the Medical Advisory Board for Digitalartforms, and shareholder in Cellectar and Elucent. Patrick R. Pfau, M.D., serves on the Scientific Advisory Board of EXACT Sciences. Jennifer M. Weiss, M.D., M.S.; Jessica R. Schumacher, Ph.D.; Aaron Potvien, M.S.; Elizabeth A. Jacobs, M.D., MAPP; and Maureen A. Smith, M.D., Ph.D., MPH, declare that they have no competing interests.

\section{Authors' Contributions}

All of the authors certify that they warrant authorship by their contribution to the (1) conception/design of the study and/or analysis/interpretation of the data, (2) writing of the manuscript, and (3) approval of the final version. The corresponding author (Jennifer M. Weiss) certifies that she had full access to all of the data in the study and takes responsibility for the integrity of the data and the accuracy of the data analysis. Jennifer M. Weiss, Jessica R. Schumacher, Maureen A. Smith, and Perry J. Pickhardt conceived the study and participated in its design and coordination. Jennifer M. Weiss, Jessica R. Schumacher, Aaron Potvien, and Maureen A. Smith performed the acquisition, analysis, and interpretation of data. Jennifer M. Weiss and Maureen A. Smith drafted the manuscript. All authors contributed to a critical revision of the manuscript for important intellectual content. Jennifer M. Weiss, Jessica R. Schumacher, Aaron Potvien, and Maureen A. Smith performed statistical analysis. All authors read and approved the final manuscript.

\section{Acknowledgments}

This work was supported by Grants R01 CA144835 and P30 CA014520 from the National Cancer Institute; Grant UL1TR000427 from the National Center for Advancing 
Translational Sciences (NCATS); Grant MRSG-13-144-01CPHPS a Mentored Research Scholar Grant in Applied and Clinical Research from the American Cancer Society; and the University of Wisconsin Health Innovation Program.

\section{References}

[1] R. Siegel, D. Naishadham, and A. Jemal, "Cancer statistics, 2013," CA Cancer Journal for Clinicians, vol. 63, no. 1, pp. 11-30, 2013.

[2] S. Winawer, R. Fletcher, D. Rex et al., "Colorectal cancer screening and surveillance: clinical guidelines and rationaleupdate based on new evidence," Gastroenterology, vol. 124, no. 2, pp. 544-560, 2003.

[3] O. Kronborg, C. Fenger, J. Olsen, O. D. Jørgensen, and O. Søndergaard, "Randomised study of screening for colorectal cancer with faecal-occult-blood test," The Lancet, vol. 348, no. 9040, pp. 1467-1471, 1996.

[4] J. S. Mandel, T. R. Church, F. Ederer, and J. H. Bond, "Colorectal cancer mortality: effectiveness of biennial screening for fecal occult blood," Journal of the National Cancer Institute, vol. 91, no. 5, pp. 434-437, 1999.

[5] J. D. Hardcastle, J. O. Chamberlain, M. H. E. Robinson et al., "Randomised controlled trial of faecal-occult-blood screening for colorectal cancer," The Lancet, vol. 348, no. 9040, pp. 14721477, 1996.

[6] P. A. Newcomb, R. G. Norfleet, B. E. Storer, T. S. Surawicz, and P. M. Marcus, "Screening sigmoidoscopy and colorectal cancer mortality," Journal of the National Cancer Institute, vol. 84, no. 20, pp. 1572-1575, 1992.

[7] J. V. Selby, G. D. Friedman, C. P. Quesenberry Jr., and N. S. Weiss, "A case-control study of screening sigmoidoscopy and mortality from colorectal cancer," The New England Journal of Medicine, vol. 326, no. 10, pp. 653-657, 1992.

[8] A. G. Zauber, S. J. Winawer, M. J. O’Brien et al., "Colonoscopic polypectomy and long-term prevention of colorectal-cancer deaths," New England Journal of Medicine, vol. 366, no. 8, pp. 687-696, 2012.

[9] R. Nishihara, K. Wu, P. Lochhead et al., "Long-term colorectalcancer incidence and mortality after lower endoscopy," The New England Journal of Medicine, vol. 369, no. 12, pp. 1095-1105, 2013.

[10] United States Preventive Services Task Force, Guide to Clinical Preventive Services, Agency for Healthcare Research and Quality, Rockville, Md, USA, 2007.

[11] B. Levin, D. A. Lieberman, B. McFarland et al., "Screening and surveillance for the early detection of colorectal cancer and adenomatous polyps, 2008: a joint guideline from the American Cancer Society, the US Multi-Society Task Force on Colorectal Cancer, and the American College of Radiology," CA Cancer Journal for Clinicians, vol. 58, no. 3, pp. 130-160, 2008.

[12] J. A. Shapiro, L. C. Seeff, T. D. Thompson, M. R. Nadel, C. N. Klabunde, and S. W. Vernon, "Colorectal cancer test use from the 2005 national health interview survey," Cancer Epidemiology Biomarkers and Prevention, vol. 17, no. 7, pp. 1623-1630, 2008.

[13] C. N. Klabunde, D. A. Joseph, J. B. King, A. White, M. Plescia, and Centers for Disease Control and Prevention (CDC), "Vital signs: colorectal cancer screening test use-United States, 2012," Morbidity and Mortality Weekly Report, vol. 62, no. 44, pp. 881888, 2013.

[14] R. Baker, J. Camosso-Stefinovic, C. Gillies et al., "Tailored interventions to overcome identified barriers to change: effects on professional practice and health care outcomes," The Cochrane
Database of Systematic Reviews, no. 3, Article ID CD005470, 2010.

[15] C. N. Klabunde, S. W. Vernon, M. R. Nadel, N. Breen, L. C. Seeff, and M. L. Brown, "Barriers to colorectal cancer screening: a comparison of reports from primary care physicians and average-risk adults," Medical Care, vol. 43, no. 9, pp. 939-944, 2005.

[16] L. C. Seeff, M. R. Nadel, C. N. Klabunde et al., "Patterns and predictors of colorectal cancer test use in the adult U.S. population," Cancer, vol. 100, no. 10, pp. 2093-2103, 2004.

[17] Z. Berkowitz, N. A. Hawkins, L. A. Peipins, M. C. White, and M. R. Nadel, "Beliefs, risk perceptions, and gaps in knowledge as barriers to colorectal cancer screening in older adults," Journal of the American Geriatrics Society, vol. 56, no. 2, pp. 307-314, 2008.

[18] A. Gilbert and N. Kanarek, "Colorectal cancer screening: physician recommendation is influential advice to Marylanders," Preventive Medicine, vol. 41, no. 2, pp. 367-379, 2005.

[19] C. P. Cairns and K. Viswanath, "Communication and colorectal cancer screening among the uninsured: data from the health information national trends survey (United States)," Cancer Causes and Control, vol. 17, no. 9, pp. 1115-1125, 2006.

[20] J. M. Weiss, M. A. Smith, P. J. Pickhardt et al., "Predictors of colorectal cancer screening variation among primary-care providers and clinics," The American Journal of Gastroenterology, vol. 108, no. 7, pp. 1159-1167, 2013.

[21] P. A. Mysliwiec, M. L. Brown, C. N. Klabunde, and D. F. Ransohoff, "Are physicians doing too much colonoscopy? A national survey of colorectal surveillance after polypectomy," Annals of Internal Medicine, vol. 141, no. 4, pp. 264-271, 2004.

[22] M. R. Nadel, J. A. Shapiro, C. N. Klabunde et al., "A national survey of primary care physicians' methods for screening for fecal occult blood," Annals of Internal Medicine, vol. 142, no. 2, pp. 86-94, 2005.

[23] L. A. Petersen, K. Pietz, L. D. Woodard, and M. Byrne, "Comparison of the predictive validity of diagnosis-based risk adjusters for clinical outcomes," Medical Care, vol. 43, no. 1, pp. 6167, 2005.

[24] A. J. Perkins, K. Kroenke, J. Unützer et al., "Common comorbidity scales were similar in their ability to predict health care costs and mortality," Journal of Clinical Epidemiology, vol. 57, no. 10, pp. 1040-1048, 2004.

[25] H. H. Pham, D. Schrag, A. S. O’Malley, B. Wu, and P. B. Bach, "Care patterns in medicare and their implications for pay for performance," New England Journal of Medicine, vol. 356, no. 11, pp. 1130-1139, 2007.

[26] National Committee for Quality Assurance, HEDIS 2012 Summary Tables of Measures, Product Lines, and Changes, National Committee for Quality Assurance, Washington, DC, USA, 2011.

[27] P. J. Pickhardt, A. J. Taylor, D. H. Kim, M. Reichelderfer, D. V. Gopal, and P. R. Pfau, "Screening for colorectal neoplasia with CT colonography: initial experience from the 1st year of coverage by third-party payers," Radiology, vol. 241, no. 2, pp. 417-425, 2006

[28] A. D. Gordon, Classification, Chapman \& Hall/CRC, Boca Raton, Fla, USA, 1999.

[29] L. Kaufman and P. Rousseeuw, Finding Groups in Data, Wiley, New York, NY, USA, 1990.

[30] S.-O. Rhee, "Factors determining the quality of physician performance in patient care," Medical Care, vol. 14, no. 9, pp. 733-750, 1976. 
[31] N. K. Choudhry, R. H. Fletcher, and S. B. Soumerai, "Systematic review: the relationship between clinical experience and quality of health care," Annals of Internal Medicine, vol. 142, no. 4, pp. 260-273, 2005.

[32] C. O'Dell and C. J. Grayson, "If only we knew what we know: identification and transfer of internal best practices," California Management Review, vol. 40, no. 3, pp. 154-174, 1998.

[33] F. J. Crosson, "The delivery system matters," Health Affairs, vol. 24, no. 6, pp. 1543-1548, 2005.

[34] J. C. Whitehead, P. A. Groothuis, and G. C. Blomquist, “Testing for non-response and sample selection bias in contingent valuation: analysis of a combination phone/mail survey," Economics Letters, vol. 41, no. 2, pp. 215-220, 1993.

[35] J.-F. Etter and T. V. Perneger, "Analysis of non-response bias in a mailed health survey," Journal of Clinical Epidemiology, vol. 50, no. 10, pp. 1123-1128, 1997.

[36] S. Barclay, C. Todd, I. Finlay, G. Grande, and P. Wyatt, "Not another questionnaire! Maximizing the response rate, predicting non-response and assessing non-response bias in postal questionnaire studies of GPs," Family Practice, vol. 19, no. 1, pp. 105-111, 2002.

[37] S. E. Kellerman and J. Herold, "Physician response to surveys: a review of the literature," American Journal of Preventive Medicine, vol. 20, no. 1, pp. 61-67, 2001. 


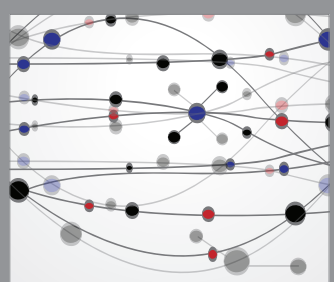

The Scientific World Journal
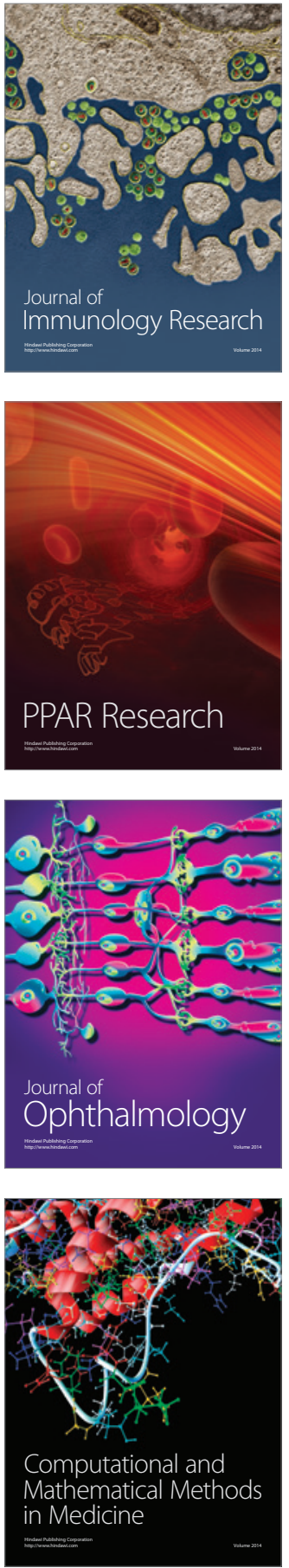

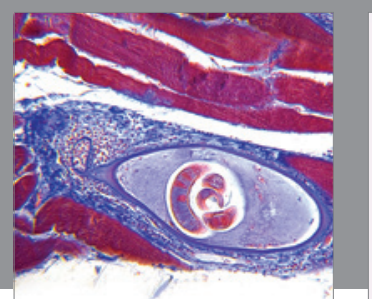

Gastroenterology Research and Practice
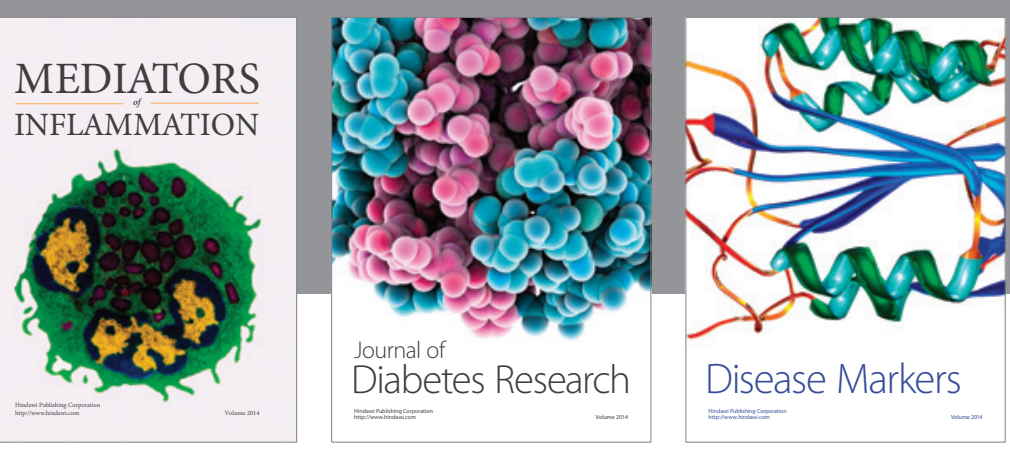

Disease Markers

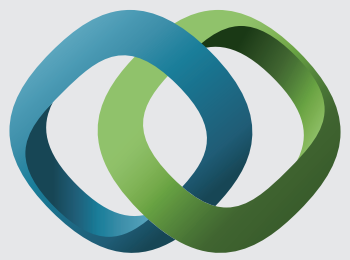

\section{Hindawi}

Submit your manuscripts at

https://www.hindawi.com
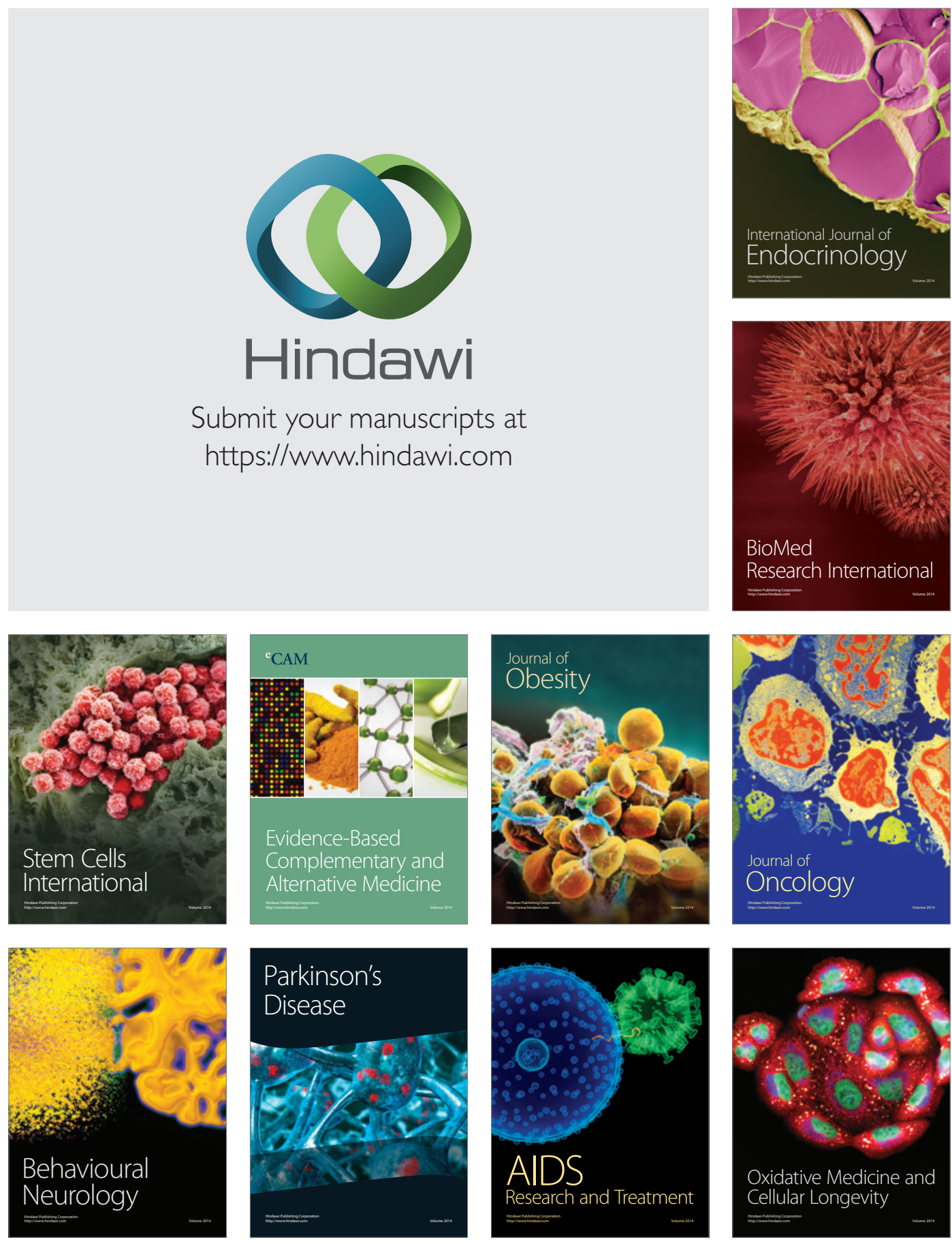